\title{
Les peuplements de Cladocères (Crustacés), descripteurs du fonctionnement hydrologique des bras-morts fluviaux ${ }^{1}$
}

\author{
C. Amoros $^{2}$ \\ D. Chessel ${ }^{3}$
}

Mots clés : écologie, Cladocères, hydrologie, hydrosystèmes, bras-morts, fleuves, méthodologie, interactions espace-temps, typologie.

J'évaluation de la valeur descriptive des peuplements de Cladocères vis-à-vis du milieu perçu comme entité spatiotemporelle (temps et espace hiérarchisés et leurs interactions) a comporté trois phases successives : classification des stations par leur fonctionnement hydrologique (origine, qualité et renouvellement de l'eau, mesurés par des paramètres phvsico-chimiques), analyse des interactions espace-temps au niveau des peuplements de Cladocères puis classification des stations par les peuplements de Cladocères. L'étude a été réalisée sur 3 bras-morts du Rhône qui diffèrent par leur mode de relation avec les eaux courantes du fleuve; chacun de ces 3 biotopes comprend plusieurs stations de mesures phusico-chimiques et faunistiques. La comparaison entre la classification hydrologique et la classification faunistique montre que les Cladocères possèdent un pouvoir descripteur fonctionnel et non stationnel ; en effet, à l'exception des grandes zones d'eau libre, profondes et dépourvues de macrophy tes, chaque peuplement transmet une information non pas sur sa station et sa végétation, mais sur son bras-mort et son fonctionnement hydrologique global.

Povulations of Cladocera (Crustacea), as describers of the hydrological functioning of stagnant waterways.

Kevwords : ecology, Cladoceran, hydrology, waterways, rivers, methodology, spatial-temporal interactions.

The descriptive value of cladoceran populations with reference to their environment is evaluated in spatial-temporal terms (time and space hierarchies and their interactions) with three successive phases : classification of stations in terms of their hydrological function (origin, quality and renewal of water, measured by physical-chemical parameters), analysis of spatial-temporal inter-actions at the level of cladoceran populations, then classification of stations in terms of their cladoceran populations. The study has been made on three stagnant sections of the Rhone, each differing in its relationship with the flowing waters of the river; each of the three biotopes had several stations at which physical-chemical and faunistic samples were taken. The comparison between the hydrological classification and the faunistic classification showed that the cladocerans had a descriptive power that was both functional and non-dependent on station ; in effect, except for the large zones of open, deep water devoid of macrophytes, each population provided information not only on the station and vegetation but also on the stagnant waterway and its total hydrological function.

\section{1. - Introduction}

\section{1. - Les bras-morts fluviaux}

Sans statut bien défini car situés à la frontière entre les eaux courantes et les eaux stagnantes, sans limites bien précises car fluctuants au gré des épisodes hydrologiques, les bras-morts des grands cours d'eau sont souvent négligés par l'écologie fluviale. Ils jouent

1. Programme Interdisciplinaire de Recherche sur l'Environne. ment (PIREN) du CNRS: * Recherches méthodologiques appliqués à la gestion écologique des systemes fluviaux a dirige par A.I. Roux.

2. Dpt. de Biol. animale et Ecologie, U.A. 367 CNRS, Université Claude Bernard, 69622 Villeurbanne Cedex France.

3. Laboratoire de Biométrie, U.A. 243 CNRS, Université Claude Bernard, 69622 Villeurbanne Cedex, France. pourtant un rôle important dans les phénomènes de régulation des peuplements fluviaux. Les bras-morts servent non seulement de zones de reproduction ou d'alimentation pour de nombreux Poissons du cours principal, mais aussi de refuges lors des grandes crues ou des pollutions accidentelles du cours d'eau (Hynes 1970, Stankovic \& Jankovic 1971, Holcik \& Bastl 1973 et 1976, Kieckhafer 1977, Roux 1984). De plus, Holden \& Green (1960), Straskraba (1965 et 1967) et Bothar (1968) ont montré que les eaux calmes des brasmorts étaient particulièrement propices à une production planctonique intense. Une grande partie de cette biomasse est très souvent entrainée dans le cours principal (Enaceanu 1964, Zivkovic 1968, Vranovsky 1973, 1974a, 1974b, Bothar 1981) et contribue par conséquent à la productivité du fleuve. 
Ces transferts de nourriture potentielle des brasmorts vers le cours principal, tout comme les déplacements de Poissons du cours principal vers les bras-morts, dépendent bien sûr de l'état et de la durée des communications entre ces deux types de bintopes. Certains bras-morts conservent une communication permanente avec le cours principal ; d'autres sont isolés chaque année lors de périodes de basses eaux; d'autres, enfin, ne sont reliés au fleuve qu'exceptionnellement, lors des grandes crues.

\section{2. - Les descripteurs de fonctionnement dans la problématique PIREN.}

L'évaluation et la prise en compte de cette diversité dans la gestion écologique des hydrosystèmes fluviaux constituent un des apports du Programme Interdisciplinaire de Recherches sur l'Environnement du CNRS (Roux \& al. 1982). Ce programme est centré sur la connaissance du fonctionnement et de l'évolution des divers écosystèmes qui composent un hydrosystène fluvial comme le Rhône. Cette recherche fondamentale a pour but finalisé l'acquisition, le traitement et la communication d'informations pertinentes pour promouvoir une véritable gestion écologique des plaines fluviales. Cette orientation vers les applications de l'écologie se traduit par la recherche de méthodologies qui puissent être mises en cuvre sur l'ensemble d'une plaine alluviale et répondre ainsi aux questions des gestionnaires.

Dans la première phase de ce programme, nous avons recherché des éléments descripteurs du fonctionnement physique, chimique et biologique des différents sous-systèmes constitutifs de l'hydrosystème. Il s'agissait de mettre en évidence des éléments de ces sous-systèmes qui intègrent les manifestations du fonctionnement du sous-systeme et donc qui peuvent transmettre une information sur ce fonctionnement, tout en réduisant le coût d'acquisition de cette information (Bournaud \& Amoros 1984).

Le travail présenté ici s'inscrit dans ce contexte: l'objectif est de savoir dans quelle mesure les peuplements de Cladocères peuvent intégrer différents fonctionnements hydrologiques de bras-morts. Ce problème peut ètre formulé en d'autres termes : une différence dans le type de relation entre un bras-mort et le cours principal du fleuve induit-elle un fonctionnement physico-chimique particulier susceptible d'influencer la structure du peuplement de Cladocères ?

\section{3. - Les peuplements de Cladocères}

Le groupe taxonomique des Cladocères a été choisi car il présente plusieurs avantages :

- les Cladocères sont de petits Crustacés particulièrement abondants dans les eaux douces calmes ou stagnantes; lẹur abondance ainsi que leur petite taille $(<5 \mathrm{~mm})$ et leurs capacités locomotrices relativement réduites, facilitent leur échantillonnage :

- leurs ceufs de durée, protégés par l'éphippie, résistent au gel, à la dessication et même au transfert dans le tube digestif de Vertébrés (Proctor 1964, Mellors 1975); ces ceufs assurent donc une tres large dissémination des espèces qui peuvent par conséquent coloniser très rapidement les milieux neufs comme les chenaux récemment abandonnés par les fleuves :

- leur mode reproduction parthénogénétique et leurs temps de génération relativement courts (de quelques jours à quelques semaines), assurent, lorsque l'environnement leur est favorable, une croissance très rapide des populations ;

- leur régime alimentaire, herbivore ou détrivore pour presque toutes les espèces, les situe à la base des réseaux trophiques et les rend donc susceptibles d'enregistrer rapidement des modifications de conditions écologiques :

- enfin, dernier avantage, pratique mais non des moindres : tous les individus capturés peuvent être identifiés sans trop de difficultés jusqu'au niveau spécifique (Amoros 1984).

\section{2. - Acquisition de l'information}

\section{1. - Les unités d'échantillonnage et l'organisa- tion de la base de données.}

Trois sites ont été choisis en fonction de leur mode de relation avec le cours principal du Rhône (un milieu sans communication avec le fleuve, un milieu en communication épisodique et un milieu en communication permanente). Sur chacun de ces sites sont localisées plusieurs stations dont le nombre total est 19 (respectivement 5, 2 et 12). A chaque station est prélevé à un instant donné un volume d'eau échantillon appelé prélèvement.

Les entités de la base de données sont donc les sites, les stations, les dates d'échantillonnage et les prélèvements dont les relations forment le plan d'échantillonnage (fig. 1a). En effet, un tel sujet de recherche hydrobiologique cons titue implicitement 
une base de données, notion qui se substitue en écologie à celle de plan d'expérience ; la figure 1 décrit la forme générale des données recueillies et l'organication de la base de données.

Chaque prélèvement se définit par son appartenance à une station et par sa date (mois, années).

Chaque station est caractérisée par :

-- son appartenance à un site géographique qui détermine son type de relation hydrique avec le cours principal du fleuve (fig. 1 b):

- l'ensemble des 5 variables qualitatives (fig. 1c).

A chaque date d'échantillonnage correspondent, pour chacune des stations : 1d) ;

- les valeurs des 5 variables quantitatives ( $f$ ig.

- les classes d'abondance des 32 espèces de Cladocères retenues pour l'analyse ( $f$ ig. 1e; Tableau I).

Tableau I. - Liste et code des espèces de Cladocères retenues pour l'analyse.

1 - Diaphanosoma brachyurum (Liévin)

2 - Sida crystallina (O.F. Müller)

3 - Ceriodaphnia laticaudata P.E. Müller

4 - Ceriodaphnia megops Sars

5 - Ceriodaphnia pulchella Sars

6. Ceriodaphnia reticulata (Jurine)

7. Daphnia ambigua Scourfield

8 - Daphnia longispina O.F. Müller

9. Daphnia pulex Leydig

10 - Scapholeberis mucronata (O.F. Müller)

11 - Simocephalis exspinosus (Koch)

12 - Simocephalus vetulus (O.F. Müller)

13 - Bosmina longirostris (O.F. Müller)

14 - Ilyocryptus agilis Kurz

15 - Acroperus harpae (Baird)

16 - Biaperiura affinis (Leydig)

17 - Alona guttata Sars

18 - Alona rectangula Sars

19 - Alonella nana (Baird)

20 - Camptocercus rectirostris Schoedler

21 - Chydorus sphaericus (O.F. Müller)

22 - Disparaloma rostrata (Koch)

23 - Eurycercus lamellatus (O.F. Müller)

24 - Graptoleberis testudinaria (Fischer)

25 - Kurzia latissima (Kurz)

26 - Leydigia quadrangtalaris (Leydig)

27 - Pleuroxus aduncus (Jurine)

28. Pleuroxus denticulatus Birge

29. Pleuroxus laevis Sars

30- Pleuroxus trigonellus (O.F. Müller)

31 - Pleuroxus truncatus (O.F. Müller)

32 - Pleuroxus uncinatus Baird

\subsection{1. - Organisation dans l'espace : sites et stations}

Les trois sites sont localisés dans la plaine alluviale du Rhône, en amont de Lyon (secteur compris entre les points-kilométriques 27 et 29 du HautRhône français).

\subsubsection{1. - Un bras-mort complètement isolé}

Les stations 1 à 5 (fig. 1b) sont situées sur un ancien méandre ("La Grande Lône-La Négria ") complètement isolé du fleuve, $y$ compris pendant les crues. Un bouchon alluvial colonisé par une végétation palustre isole pendant une grande partie de l'année les stations 1 et 2 (secteur amont) des stations 3,4 et 5 (secteur aval) ; le secteur amont parait beaucoup plus atterri et plus eutrophe que le secteur aval.

\subsubsection{2. - Un bras-mort connecté lors des crues}

Les stations 6 et 7 correspondent à la partie amont ("Les Mouilles ") d'un ancien chenal du Rhône ; elles ne sont en contact direct avec les eaux fluviales que lors des crues; leur alimentation est assurée en grande partie par les eaux interstitielles du sousécoulement du Rhône (Reygrobellet \& al. 1981).

\subsubsection{3. - Un bras-mort en communication permanente}

Les stations 8 à 19 s'échelonnent sur la partie aval du même ancien chenal qui forme la « Lône des Pêcheurs "; ce bras-mort fermé à l'amont, conserve une communication permanente avec le fleuve par son extrémité aval. De ce fait, il reçoit deux types d'eaux : les eaux interstitielles du sous-écoulement du Rhône qui diffusent à travers les alluvions grossières déposées à l'amont et les eaux fluviales qui refluent par l'aval. L'importance relative de ces deux alimentations est déterminée par les fluctuations du régime du Rhône puisque le débit du sousécoulement dépend lui-même du débit du fleuve (Juget \& al. 1979). Ainsi par très basses eaux, lorsque le débit du fleuve est localement inférieur à 250 $\mathrm{m}^{3} . \mathrm{s}^{-1}$. il $\mathrm{n}^{\prime} \mathrm{y}$ a pas de mise en charge du sousécoulement ; pendant ces étiages, le barrage hydroélectrique, situé à $500 \mathrm{~m}$ en aval de la confluence entre le bras-mort et le fleuve, maintient un niveau relativement élevé et provoque un reflux d'eaux fluviales sur l'ensemble du bras-mort. En revanche, lorsque le débit du Rhône dépasse $250 \mathrm{~m}^{3} . \mathrm{s}^{-1}$, le sous écoulement est mis en charge et les caux souterraines s'écoulent dans le bras-mort. 
a - Plan d'EChantuloninage

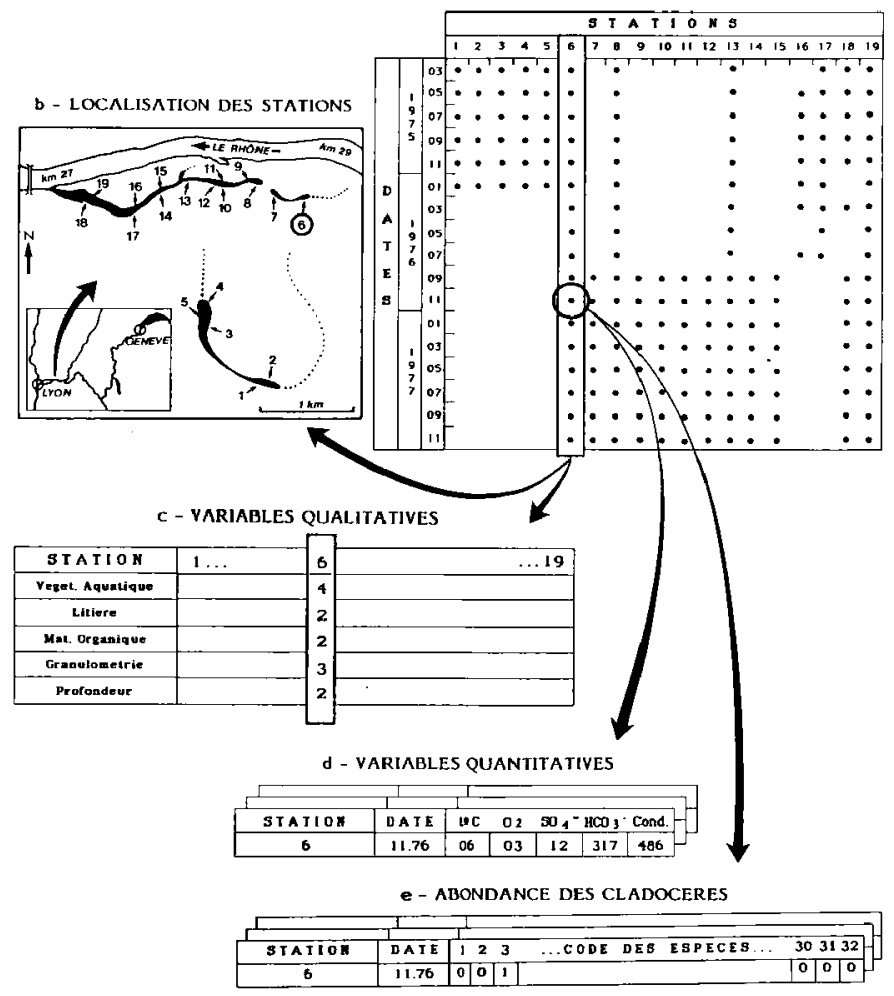

Fió. 1: Organisatiun de la base de données : le plan d'échantillonnage (a) schématise les relaions entre les ęnités (sites, stations, dates d'échantillonnage) dont les attributs sont les paramètres stationnels (c), les paramètres associés à chaque prélèvement (d) et l'abondance des espèces de Cladocères dans chaque prélèvement (e). 


\subsection{2. - Organisation dans le temps : un plan d'échantil- lonnage non orthogonal.}

Le schéma d'organisation de la base de données (fio. 1a) révèle un plan d'échantillonnage non orthogonal. Les mesures de variables quantitatives et les prélèvements de Cladocères ont été réalisés tous les deux mois pendant trois ans $(1975,1976,1977)$ mais il s'agissait de deux campagnes successives et seules 5 stations ont été suivies régulièrement pendant toute la durée des 2 campagnes ; 7 stations ont été échantillonnées pendant la première campagne seulement et 7 nouvelles stations ne l'ont été que pendant la deuxième campagne (fig. 1a).

La réduction du coût d'acquisition de l'information écologique constituant l'un des objectifs de notre programme de recheche PIREN, la première phase de ce programme s'appuie donc sur l'exploitation de données déjà disponibles, recueillies pour d'autres objectifs. Malgré ses inconvénients, cette procédure présente un intérêt méthodologique ; il est en effet fréquent en écologie appliquée, notamment lors d'études d'impact de projets d'aménage. ments, d'être confrontè à ce genre de problème : l'utilisation de données préexistantes. La question qui se pose alors est la suivante : quelles techniques de traitement de données peut-on utiliser face à un plan d'échantillonnage déséquilibré vis-à-vis de l'ubjertif poursuivi, ou face à des données manquantes ?

\section{2. - Les mesures}

2.2.1. - Les paramètres associés à chaque station

Chaque station est caractérisée par 5 variables qualitatives ( $f$ ig. $1 \mathrm{c}$ ).

2.2.1.1. - La végétation aquatique est exprimée par 5 modalités :

1 = eau libre dépourvue de macrophytes:

2 = phragmitaie aquatique (Scirpeto Phrag. mitetum) ;

$3=$ herbiers du Myriophyllo-Nupharetum;

$4=$ herbiers d'hydrophytes divers;

$\mathbf{5}=$ macrophytes à feuilles flottantes en été seulement (Potamogeton natans p. ex).

2.2.1.2. - La litière de débris organiques est exprimée par 3 modalités :

$1=$ litière absente;

2 = litière présente :

$3=$ litière abondante.
2.2.1.3. - La charge en matière organique des sediments superficiels est exprimée par 3 modalités en fonction des concentrations en carbone organique :

$1=$ concentrations inférieures à $2 \%$;

$2=$ concentrations comprises entre 2 et $4 \%$;

$3=$ concentrations comprises entre 4 et $8 \%$ :

2.2.1.4. - La texture des éléments superficiels est exprimée par 3 modalités :

$1=$ argiles et limons fins;

$2=$ limons sableux ;

$3=$ sables.

2.2.1.5. - La protondeur est elprimée par 4 classes qui intègrent les fluctuations dans le temps du niveau de l'eau:

$1=0,2$ à $0,7 \mathrm{~m}$

$2=0,4$ à $1,1 \mathrm{~m}$

$3=1,0$ a 2,0

4 = supérieure à $2,5 \mathrm{~m}$

2.2.2. - Les paramètres associés à chaque prélèvement

Les bras-morts sont soumis à deux rythmes principaux : le débit du fleuve réglant les apports d'eau éventuels et le rythme saisonnier se manifestant notamment par des variations de température. Le premier est un processus à variations fortes, brutales et d'apparence aléatoire (impact des barrages hydroélectriques) alors que le second est strictement périodique.

Pour percevoir le fonctionnement hydrologique de chaque station, 6 paramètres physico-chimiques ont été retenus; il s'agit de la température de l'eau, de l'oxygène dissous, de la hauteur d'eau, de la conductivité électrique, de la teneur en bicarbonates (très abondants dans les eaux interstitielles du sousécoulement) et de la teneur en sulfates (très abondants dans les eaux superficielles du Rhône). Les mesures et les prélèvements d'eau pour les dosages chimiques, ont été effectués à $-0.5 \mathrm{~m}$ de profondeur.

\subsection{3. - Les paramètres faunistiques}

Dans les stations de faible profondeur (jusqu'à 1,3 $\mathrm{m})$, les échantillonnages ont été effectués à l'aide d'un tube qui prélève des colonnes d'eau de $20 \mathrm{~cm}^{2}$ de section. de la surface jusqu'au fond (Amoros 1980). De 1 à 3 prélèvements ont été réalisés à chaque station. En eau libre, à plus grande profondeur, 
le tube ne pouvant être manipulé efficacement à partir d'une embarcation, nous avons utilisé une bouteille de Van Dorn qui, immergée verticalement, prélève une colonne d'eau de $1 \mathrm{~m}$. correspondant à un volume de 7 litres. Dans chaque station d'eau libre ont été réalisés deux prélèvements, un près de la surface et l'autre près du fond. Les volumes d'eau ainsi prélevés sont filtrés sur des tamis en nylon de $0,2.30 \mathrm{~mm}$ de vide de maille.

Les effectifs de chaque espèce sont exprimés en classes d'abondance dont les limites sont définies par une progression géométrique de raison 4,3 (Frontier 1969) :

Classe 1 : de 1 à 3 individus. $\mathrm{I}^{-1}$ ou. $10 \mathrm{~cm}^{-2}$ Classe 2 : de 4 à 17 individus. $1^{-1}$ ou. $10 \mathrm{~cm}^{-2}$ Classe $3:$ de 18 à 80 individus. $1^{-1}$ ou. $10 \mathrm{~cm}^{-2}$ Classe $4:$ de 81 à 350 individus. $\mathrm{I}^{-1}$ ou. $10 \mathrm{~cm}^{-2}$ etc...

Frontier \& Ibanez (1974) ont montré les avantages pratiques de ce mode d'expression des données qui ne modifie pratiquement pas l'information transmise sur la structure des peuplements.

Cinq espèces de Cladocères ont été exclues de l'analyse car elles ne sont présentes qu'une seule fois dans une seule station; il s'agit de :

- Ceriodaphnia quadrangula (O.F. Müller);

- Lathonura rectirostris (O.F. Müller);

- Alona quadrangularis (O.F. Müller);

- Alonella exigua (Lilljeborg);

- Oxyurella tenuicaudis (Sars).

\section{3. - Traitement de l'information et interprétation des résultats}

L'objectif principal d'une telle expérience est de préciser la valeur descriptive du groupe taxonomique étudié en relation avec le milieu perçu comme entité spatio-temporelle (temps et espace hiérarchisés et vraisemblablement interactions). On peut affirmer qu'aucune procédure automatique de nature statistique ou informatique ne permet actuellement de résoudre un tel problème; l'analyse des données a donc comporté trois phases successives : classification des stations par leur fonctionnement hydrologique, analyse des interactions espace-temps au niveau des peuplements de Cladocères et classi. fication des stations par les peuplements de Cladocères.

\section{1. - Classification des stations par le fonction- nement hydrologique.}

Nous avons d'abord examiné la nature des variations physico-chimiques en liaison avec l'espace perçu au niveau de la station et au niveau du site. Les données brutes forment un processus multiva. rié (6 variables). L'analyse graphique discutée par Auda (1983) permet d'établir une typologie de fonctionnement (fig. 2).

Les types A (stations 1 et 2) et B (stations 3, 4 et 5) correspondent au type paléopotamon défini par Roux \& al. (1982); il s'agit d'anciens chenaux méandriformes, complètement isolés du fleuve, aux eaux stagnantes d'origine essentiellement plurviale (variations concordante des teneurs en sulfates et bicarbonates). Le type A est beaucoup plus eutrophe et plus at terri que le type $B$; cette différence se manifeste notamment sur les variations des teneurs en oxygène dissous (fig. 2).

Le type $\mathrm{C}$ (stations 6 et 7 ) correspond au plésiopotamon c'est-à-dire à des eaux stagnantes très peu profondes envahies par les macrophytes aquatiques (fortes variations des teneurs en oxygène dissous). L'origine de l'eau est le sous-écoulement fluvial (variations relativement faibles des teneurs en sels dissous) mais ces biotopes sont submergés chaque année, pendant de très courtes périodes, par les eaux fluviales en crue.

Le type D (stations 8 à 19) correspond au parapotamon c'est-à-dire à un bras-mort qui conserve à l'aval une communication permanente avec le fleuve. Ce type de bras-mort reçoit deux sortes d'eaux : Ies eaux souterraines du sous-écoulement qui diffusent à l'amont et les eaux superficielles fluviales qui refluent par l'aval. Cette double origine de l'eau se traduit par la discordance des variations des teneurs en sulfates et en bicarbonates car les eaux superficielles fluviales sont très chargées en sulfates tandis que les eaux souterraines du sousécoulement sont très chargées en bicarbonates. L'affrontement de ces deux masses d'eau induit l'existence d'un gradient physico-chimique le long du bras-mort. Le type D peut être subdivisé en 3 soustypes qui traduisent l'existence de ce gradient : D1 dans la partic amont (stations 8 à 12), D2 dans la partie médiane (stations 13 à 15) et D 3 dans la partie aval (stations 16 à 19). Le type $D$ décrit donc une masse d'eau sans cesse renouvelée, soumise à d'incessants mouvements de flux et de reflux qui se 

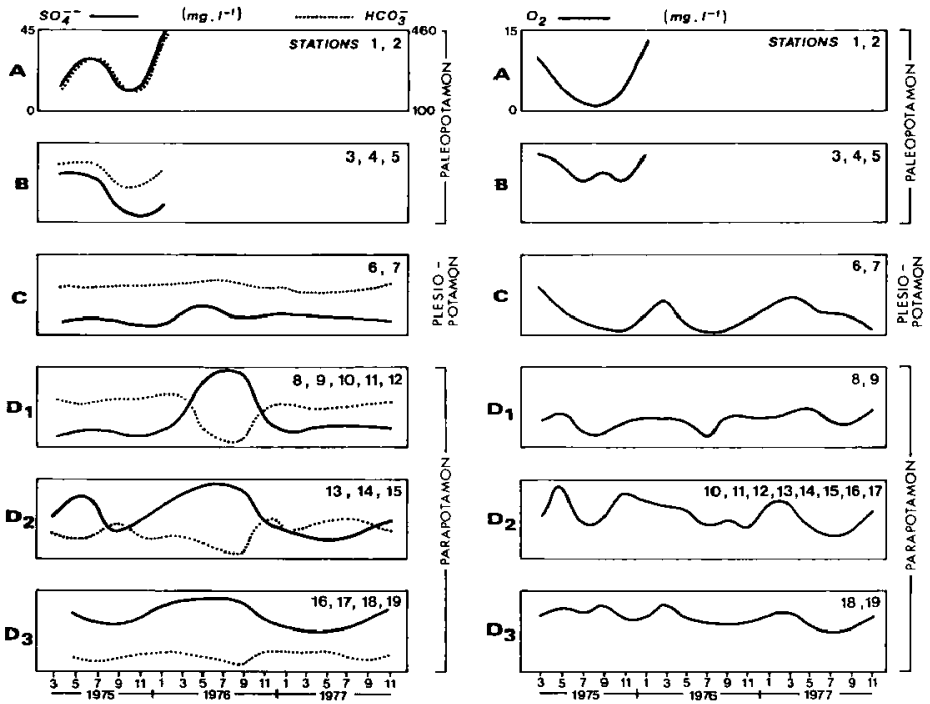

Fio. 2: Typologie functionnelle établie à partir des fluctuations de 3 variables quantitatives: les tenturs cn sultates, en birarbonates et en oxygène dissous ; la nomenclature indiquée en marge (paléopotamon, plésiopotanıon et parapotamon) a été définie par Roux \& al. (1982)

manifestent par des courants d'équilibrage ; ces courants sont néanmoins bien trop faibles pour être visuellement perceptibles sauf à la station 13 du fait du rétrécissement du chenal.

Pour les phases suivantes du traitement des données, les paramètres physico-chimiques associés aux prélèvements pourront donc être résumés par cette tynologie de fonctionnement ; les analyses ultérieures porteront alors sur un tableau de 7 variables qualitatives associées à chaque station (Tableau II) parmi lesquelles figurera le fonctionnement hydrologique $(6$ modalités correspondant aux 3 types +3 sous-types). La hauteur d'eau dont $96 \%$ de variance expliquée traduisent sa liaison très étroite avec les stations (1\% de variance expliquée par le temps), sera aussi traitée comme variable stationnelle.

\section{2. - Analyse des interactions espace-temps au niveau des peuplements de Cladocères}

La seconde phase du traitement des données a cherché à préciser dans le tableau faunistique (185 prélèvements, 32 taxons) l'influence respective de la station et de la date d'échantillonnage. Le tableau faunistique est dépouillé par une analyse factorielle des correspondances (AFC). La représentation fonctionnelle des trois premiers facteurs de l'AFC (fig. 3) montre que la structure faunistique est d'abord descriptrice des stations. En effet, on peut considérer la coordonnée factorielle comme la moyenne d'un code numérique affecté à chaque espèce, dont la valeur discriminante est optimale (Estève 1978). Si les rythmes saisonniers s'expriment par l'évolution des coordonnées factorielles sur les 3 premiers 
Tableau II. - Variables stationnelles: les variables 1 et 2 (respectivement le "fonctionnement en $\mathrm{O}_{2}$ " et le * fonctionnement hydrique ) expriment la synthèse de variables quantitatives (voir texte $: 3.1 \mathrm{et}$ fig. 2 ) avec les modalités suivantes : $1=$ fonctionnement de type $A$, 2 = type B, $3=$ type C, $4=$ type D1, $5=$ type D2, 6 $=$ type D3) ; les modalités des variables 3 à 7 sont définies dans le texte (2.2.1).

\begin{tabular}{|c|c|c|c|c|c|c|c|}
\hline et et iores & $\begin{array}{l}\text { fonction. } \\
\text { or }\end{array}$ & $\begin{array}{l}\text { fonction. } \\
\text { hydricave }\end{array}$ & $\begin{array}{l}\text { vegt. } \\
\text { equat. }\end{array}$ & Itibre & $\begin{array}{l}\text { mat. } \\
\text { orga. }\end{array}$ & grereulo. & profond. \\
\hline 1 & 1 & 1 & 2 & 3 & 3 & 1 & $\downarrow$ \\
\hline 2 & 1 & 1 & 5 & 2 & 3 & 1 & 3 \\
\hline 3 & 2 & 2 & 3 & 2 & 3 & 1 & 1 \\
\hline 4 & 2 & 2 & 3 & 3 & 3 & 1 & 2 \\
\hline 5 & 2 & 2 & 1 & 1 & 3 & 1 & 4 \\
\hline 6 & 3 & 3 & $a$ & 2 & 2 & 3 & 2 \\
\hline 7 & $y$ & 3 & 3 & 3 & 3 & 1 & 2 \\
\hline$\theta$ & 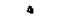 & 4 & 5 & 1 & 2 & 3 & 2 \\
\hline 9 & 4 & 4 & 3 & , & 3 & 2 & 2 \\
\hline 10 & 5 & 4 & 3 & 2 & 3 & 1 & 2 \\
\hline 11 & 5 & 4 & 5 & $z$ & 3 & 1 & 2 \\
\hline 12 & 5 & 4 & 1 & 2 & 3 & 1 & 3 \\
\hline 13 & 5 & 5 & 2 & 2 & 3 & 2 & 2 \\
\hline 14 & 5 & 3 & 2 & 2 & 2 & 1 & 2 \\
\hline 15 & 5 & 5 & 1 & 1 & 2 & 1 & 4 \\
\hline 16 & 5 & 6 & 2 & 2 & 3 & 1 & 2 \\
\hline 17 & 5 & 6 & 1 & 1 & 2 & 1 & 4 \\
\hline IB & 6 & 6 & 2 & 2 & 3 & 1 & 1 \\
\hline 19 & 6 & 6 & 1 & 1 & $d$ & 2 & 3 \\
\hline
\end{tabular}

farteurs de l'AFC (fig. 3) on remarquera cependant que la maniere dont ces rythmes s'expriment (valeurs positives ou négatives des coordonnées et forme des graphes) permet de discriminer d'abord des stations ou des groupes de stations (voir par exemple les stations 1 et $2 ; 6$ et $7 ; 9,10,16$ et 18 ).

Une appréciation d'ensemble peut être portée sur la nature de la variation de la liste faunistique. Le tableau III montre les liaisons entre d'une part les coordonnées factorielles des prélèvements et d'autre part les variables enregistrées sur ceux-ci. La liaison avec la variable qualitative \& station" ou " rate " est mesurée par un rapport de corrélation (pnurcentage de variance expliquée par la répartition en classes). La liaison avec l'une des variables physico-chimiques est mesurée par un coefficient de corrélation linéaire.

Les interactions stations $x$ dates sont en première analyse secondaires; la structure spatiale l'emporte largement sur la structure temporelle.

Les résultats de cette analyse préliminaire nous autorisent à simplifier le tableau faunistique en le résumant au plan stationnel par le calcul des
Tableau III. - Analyse préliminaire des liaisons entre le contenu faunis tique (codé numériquement par les facteurs de l'A.F.C.) et d'une part la répartition spatiotemporelle des prélèvements et d'autre part les variables de milieu mesurées.

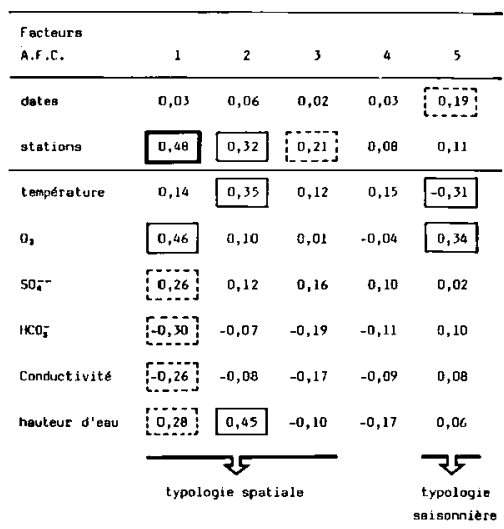

relevés moyens par station. La troisieme phase de l'analyse des données traitera ce tableau faunistique résumé.

\section{3. - Classification des stations par les peuple- ments de Cladocères}

Le tableau faunistique de relevés moyens par station est soumis à l'analyse factorielle des correspondances et à la classification automatique. Cette pratique, d'usage assez général, impose cependant le modèle de la partition des relevés sans toutefois le justifier pleinement. Nous présentons, comme c'est l'usage, la carte factorielle $\mathbf{F} 1 \times \mathbf{F} 2$ avec l'expression de la classification automatique (fig. 4).

Nous projetons sur cette carte l'abondance de chaque espèce représentée par un carré dont la surface est proportionnelle à cette abondance. A titre d'exemple les cartes obtenues pour quelques espèces sont reproduites sur la figure 5 .

La distribution de chacune des 32 espèces dans les groupes de stations définis par la procédure de classification automatique détermine la réécriture du tableau de données faunistiques (fig. 6). Ce tableau valide et décrit complètement l'interprétation exécutée. 

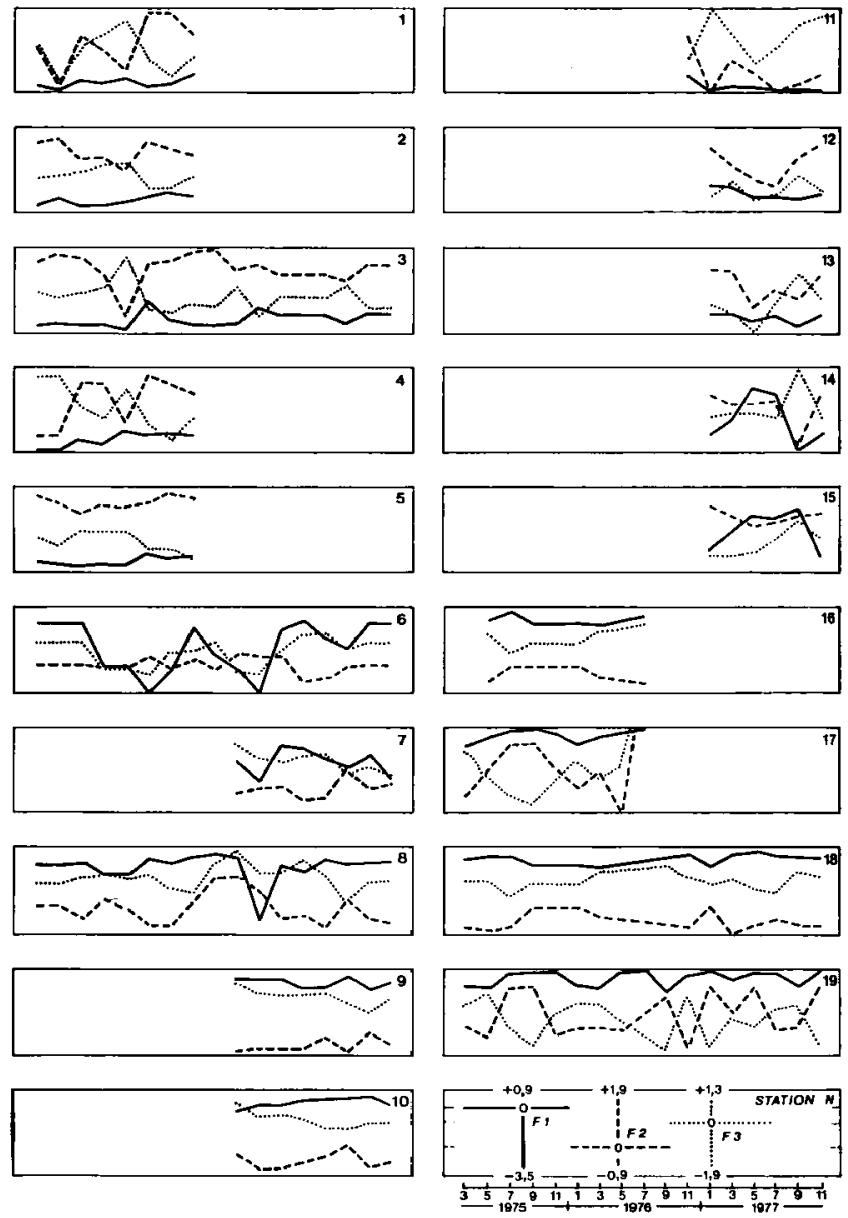

Fiq. 3 : Représentation fonctionnelle des 3 premiers facteurs de l'analyse factorielle des correspondances des relevés faunistiques en fonction du temps pour chacune des 19 stations. 


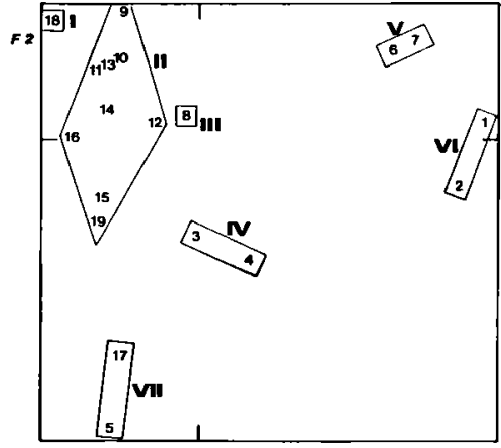

$F_{1}$

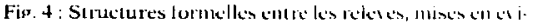
dence par l'analyse statistique des peuplements de Cladocères (plan F1 $\times$ F2 de l'A.F.C.).

\section{4. - Discussion}

Si on projette sur l'expression de la classification des stations par les peuplements de Cladocères ( $f$ ig. 4). la typologie fonctionnelle établie à partir des variables hydrologiques (fig. 2), on constate une bonne correspondance entre les deux typologies $(f \mathrm{ig}$. 7). Les autres variables stationnelles (végétation aouatique, litière de débris organiques, teneur en matière organique et texture des sédiments superficiels, hauteur d'eau) ne montrent aucune correspondance avec la classification des stations obtenue à partir des communautés de Cladocères. Pour mesurer l'accord entre deux classifications on utilise le test dérivé du coefficient d'autocorrélation spatiale de Cliff \& Ord (1973) qui s'appuie sur le nombre de paires de points apparues dans la même classe de chacune des deux classifications (Tableau IV.

Les variations des structures faunistiques tradujsent donc la typologie de fonctionnement hydrologinue. Les peuplements de Cladocères expriment ceoendant une partition plus fine que celle foumie par le seul traitement des variables abiotiques. En effet. parmi l'ensemble des stations localisées sur le bras-mort identifié comme parapotamon (fonctionnement de type D), 3 stations s'individualisent : il s'agit des stations 8 et 18 d'une part et de la station 17 d'autre part. L'interprétation écologique de cette nouvelle partition est relativement aisée.

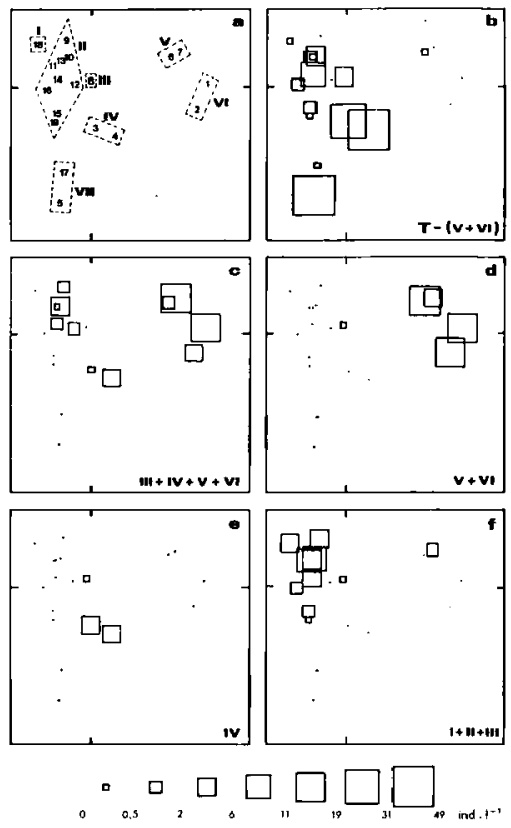

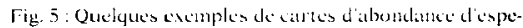
ces projetées sur le plan F1 $\times$ F2 de l'A.F.C. :

$\mathbf{a}=$ classification automatique des stations sur F1 $\times$ F2;

$\mathrm{b}=$ abondance de Ceriodaphnia pulchella, espèce héléoplanctonique, présente dans toutes les stations sauf dans les groupes V et VI ;

$c=$ abondance de Simocephalus exspinosus, espèce phytophile :

$\mathrm{d}=$ abondance de Daphria pulex, espece correspondant aux fonctionnements de type $\mathrm{A}$ et $\mathrm{C}$;

$e=$ Pletroxus laevis, espece correspondant au fonctionnement de type B ;

$\mathrm{f}=$ Pleuroxus uncinatus, espèce correspondant au fonctionnement de type $\mathbf{D}$ :

* les carrés représentent les classes d'abondance moyenne par prélèvement.

(Logiciel graphique de Auda 1985).

Les stations 8 et 18 correspondent aux 2 extrémités du parapotamon "La Lône des Pêcheurs". La station 8 est localisée à l'extrémité amont ; c'est la seule station de parapotamon établje sur un substrat sableux à travers lequel diffusent les eaux interstitielles du sous-écoulement qui alimentent le 


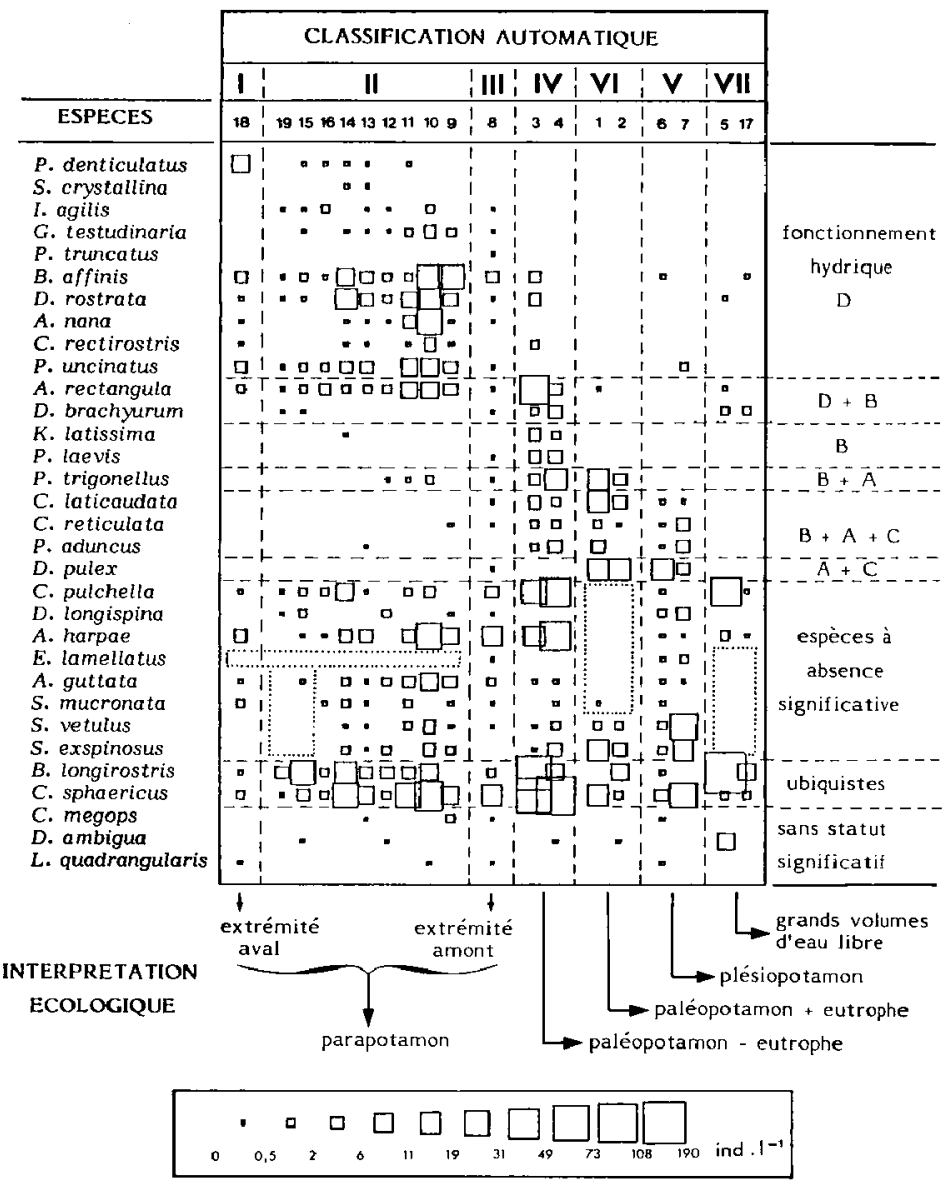

Fig. 6: Abondance des espèces de Cladocères dans chaque station et interprétation écologique (cf. fig. 7) : tableau de données réoganisé a partir de l'analyse factorielle des correspondances et de la procedure de classification au tomatique (Logiciel graphique de Auda 1985). 


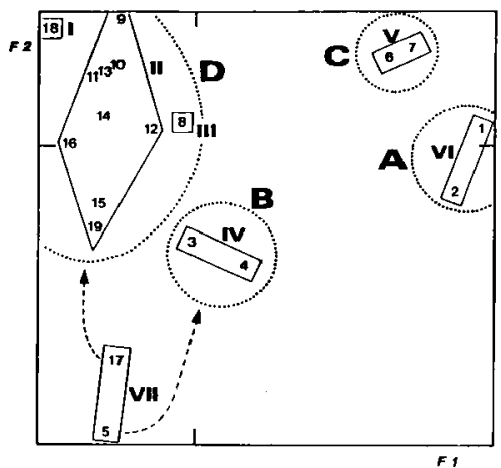

Fir. 7 : Projection de la typologie fonctionnelle (en pointillés) produite par les variables quantitatives de milieu (cf. fig. 2), sur l'expression de la classification automatique des stations (en traits pleins) produite par les peuplements de Cladocères (cff fig. 4);

Tableau IV. - Comparaison de la classification des stations obtenue à partir des communautés de Cladocères avec celle obtenue à partir des variables stationnelles (test dérivé du coefficient d'autocorrélation spatiale de Cliff \& Ord 1973).

\begin{tabular}{|c|c|c|}
\hline variableg olotionnelles & $\begin{array}{l}\text { prababjluté de } \\
\text { depassar l observation }\end{array}$ & $\begin{array}{l}\text { correapondence } \\
\text { guec } \\
\text { 1a clasification } \\
\text { des Clodoceres }\end{array}$ \\
\hline "fonct ionmenent 02" & $<10^{-4}$ & treo significative \\
\hline "ronct i onnement hydrique" & $<10^{-4}$ & tres aigaificat ive \\
\hline pro rondeur & 0,07 & ces limite \\
\hline voptetation acwatique & 0,10 & non \\
\hline texture deg ef́diments & 0,14 & non \\
\hline Jitiere de debcia orga. & 0,29 & non \\
\hline mat. orge. des sédtimenty & 0,86 & non \\
\hline
\end{tabular}

bras-mort . La station 18 est située à l'extrémité aval du parapotamon, dans une phragmitaie très peu profonde (c'est la station la moins profonde de ce parapotamon). Outre les phénomènes d'exondation auxquels elle est périodiquement soumise - les échantillons manquants ( $f i g$. i a) correspondent à ces phases d'exondation - la station 18 subit probablement l'effet de la proximité du Rhòne en recevant notamment les alluvions limoneuses transportées par les eaux fluviales qui refluent. Cette station abrite moins d'espèces que les autres stations du bras-mort (fio. 6).
La station 17 est perçue par les Cladocères (fig. 4) comme relativement proche de la station 5 elleméme située sur un autre bras-mort, de type paléopotamon (fonctionnement de type B). Bien qu'appartenant à 2 bras-morts géographiquement distincts et aux fonctionnements hydriques différents, ces 2 stations ont une caractéristique commune, la présence d'un grand volume d'eau libre dépourvu de macrophytes; il s'agit en effet des stations les plus profondes et les plus larges des 3 sites explorés (station 17 : profondeur $3 \mathrm{~m}$, largeur $40 \mathrm{~m}$, ; station 5 : profondeur $4,8 \mathrm{~m}$, largeur $90 \mathrm{~m}$ ). La présence d'eau libre profonde constitue un caractère écologique plus déterminant que le type de fonctionnement hydrique. Ceci s'explique par le fait que ces grands volumes d'eau libre dépourvus de macrophytes sont défavorables aux especes phytophiles qui constituent l'essentiel du peuplement de Cladocères des bras-mort fluviaux, et n'abritent surtout que des espèces héléoplanctoniques.

L'examen des groupements d'espèces révèle quelques similitudes entre le groupe des stations 5 et 17 et les stations 15 et 19 (fig. 6). La station 15, dépourvue de macrophytes, est aussi profonde que la station 17 mais beaucoup plus étroite ( $10 \mathrm{~m}$ de large) ce qui l'expose à une contamination à partir des communautés des herbiers littoraux. La station 19, elle aussi dépourvue de macrophytes, est très large (60 $\mathrm{m}$ de large) mais sa profondeur n'atteint pas 2 $m$ et ne constitue donc pas un biotope très favorable aux espèces héléoplanctoniques.

En examinant le tableau de données réorganisé en fonction de la répartition des espèces (fig. 6), on constate que les groupes de stations ont une définition multispécifique; il y a très peu d'espèces descriptives strictes mais des groupes d'espèces descriptives d'un type de fonctionnement. On peut tenter de modéliser ce tableau en le simplifiant. D'une manière générale, un tableau de données especes $x$ relevés (fig. 8) peut être réorganisé soit sous la forme A (dans le cas d'une structure en gradient ou en écailles) soit sous la forme $B$ (mise en évidence d'associations). Dans le cas présent, on chercherait en vain une réorganisation pertinente sous une forme A ou B. Le groupe taxonomique des Cladoceres présente ici de fortes structures écologiques non réductibles à une simple ordination ou une simple classification. La forme générale de ce genre de dispersion d'espèces peut être schématisée par la figure 9. Les groupes d'espèces ainsi définis, correspondent à 

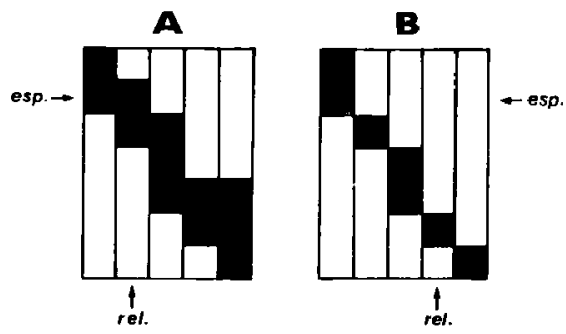

Fì, 8: Formes générales de réorganisation d'un lableaz de données espèces $\mathrm{x}$ relevés :

$A=$ gradient, écailles;

$\mathbf{B}=$ associations :

esn. = groupes d'espèces ;

rel. = groupes de relevés.

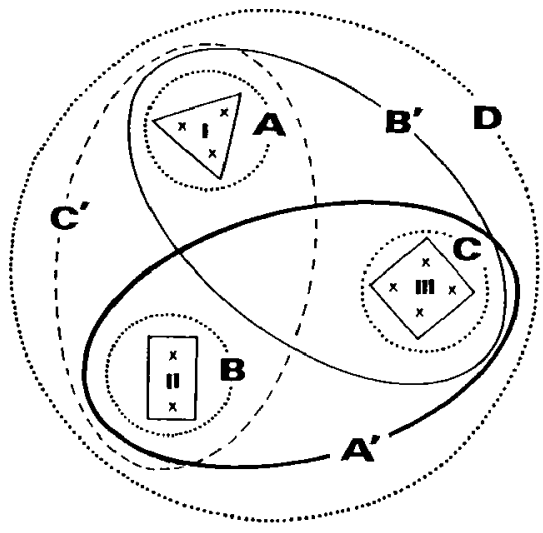

Fir. 9 : Forme générale de la dispersion des espèces dans la présente étude:

I, II, III = groupes de relevés semblables au plan faunistique:

A, B, C = groupes d'espèces indicatrices:

$A^{\prime} . B^{\prime}, C^{\prime}=$ groupes d'espèces à absence indicatrice ;

$\mathbf{D}=$ espèces ubiquistes. des niveaux hiérarchiques de l'influence des variables du milieu (par exemple, le caractère « fonctionnement du bras-mort " est plus puissant que les caractères propres à chaque station). L'analyse de ce type de structures nécessite des moyens d'analyse des données propres au domaine hydrobiologique.

\section{5. - Conclusion}

Si on s'en tient à des prélèvements en eau de profondeur limitée, les peuplements de Cladocères décrivent non pas la station et sa végétation, mais les différents bras-morts et leur fonctionnement hydrique global. Ce groupe taxonomique possède donc une valeur descriptive fonctionnelle et non stationnelle. Ce pouvoir descripteur fonctionnel atteint cependant ses limites dans les grandes zones d'eau libres dépourvues de macrophytes. En effet, les peuplements de Cladocères de ce type d'habitat sont consitués d'espèces héléoplanctoniques relativement ubiquistes et sont en fait caractérisés par l'absence des espèces phytophiles qui constituent l'essentiel du peuplement de Cladocères des bras-morts.

Hormis la hauteur d'eau, les variables stationnelles (macrophytes aquatiques, teneur en matière organique et texture des sédiments superficiels, litière de débris organiques) paraissent ici pratiquement sans importance mais il existe des interactions dans la mesure où toutes les combinaisons " type de bras-mort $x$ type d'herbier aquatique "n'ont pas été échantillonnées au cours de ce travail ou n'existent pas.

Exprimées par leur peuplement de Cladocères, deux stations d'un même bras-mort apparaissent toujours plus similaires que deux stations situées dans le même type d'herbier dans deux bras-morts différents.

Remerciements

Ce travail a été réalisé dans le cadre du Programme Interdisciplinaire de Recherche sur J'Environnement (PIREN du CNRS) a Recherches méthodologiques appliquées à la gestion écologique des ressources en eau dans les systèmes fluviaux » dirigé par A.L. Roux et soutenu par la Commission des Communautés Européennes (contrat EN-693-F/5D), La première série de données a été recueillie lors de la réalisation d'une convention avec le Ministère de l'Environnement (Comite " Faune et Flore *, convention $n^{0}$ 76-55). Les analyses physico-chimiques de l'eau ont été réalisées par l'équipe * Lônes * du Groupe de Recherches sur le Haut-Rhône Francais (U.A. 367 CNRS). 


\section{Travaux Cités}

Amoros (C.). 1980. - A simple device for quantitative pseudoperiphyton sampling. Hydrobiologia, 68 (3) : 243-246.

Amoros (C.). 1984. - Introduction pratique à la systématique des organismes des eaux continentales françaises. 5 . Crustacés Cladocères. Edit. Assoc.Franç. Limnol., Paris, 63 p.

Aurla (Y.). 1983. - Róle des méthodes graphiques en analyse des données : application au dépouillement des enquêtes écologiques. Thèse 3 e cycle, Lyon : $127 \mathrm{p}$.

Aurla (Y.). 1985. - Logiciel graphique pour l'analyse des données (Fortran 77) - Laboratoire de Biométrie, Lyon, Ronéo: 107 p.

Bothar (A.) 1968. - Untersuchungen des Donauplankions an Entomostraca während der grossen Uberschwemmung im Jahre 1965 (Danub. Hung XL VIIT). Ann Univ. Sci. Budapest, sect. Biol., 9-10:87-98.

Bothar (A.). 1981. - Vergleichende Untersuchung der Crustacea Gemeinschaften in Nebenarm * Alte Donau * und im Haupts. trom (Stromkm 1481) (Danub. Hungar XCIV). Ann. Univ. Sci. Budapest, sect. Biol., 22-23: 159-174.

Bournaud (M.) \& Amoros (C.). 1984. - Des indicateurs biologiques aux descripteurs de fonctionnement : quelques exemples dans un systeme fluvial. Bull. Ecol., 15 (1): 57-66.

Cliff (A.D.) \& Ord (J.K.). 1973. - Spatial autocorrelation. Pion, London, 125 p.

Enaceanu (V.). 1964. - Das Donauplankton auf rumänischen Gebiet. Arch. Hydrobiol., suppl., 27 : 442-456.

Estève (J.). 1978. - Les méthodes d'ordination : éléments pour une discussion. In : Legay J.M. et Tomassone R. (Eds) Biométrie et écologie, Soc. Fr. Biom, Paris, 223-250.

Frmtier (S.). 1969. - Sur une methode d'analyse faunistique rapide du zooplancton. J. Exp. Mar. Biol. Ecol., 3 (1) : 18-26.

Fmntier (S.) \& Ibanez (F.). 1974. - Utilisation d'une cotation d'abondance fondée sur une progression géométrique pour l'analyse des composantes principales én écologie planctonique. J. Exp. Mar. Biol. Ecol., 14 (3) : 217-224.

Holcik (J.) \& Bast! (I.). 1973. - Ichthyocenoses of two arms of the Danube with regard to changes in species composition and population density in relation to the fluctuations of the water level in the main stream. (En slovaque, rés. angl.), Biol. Prace, $19(1): 5-106$.

Holcik (J.) \& Bastl (L.). 1976 . - Ecological effects of water level fluctuation upon the fish populations in the Danube River flood plain in Czechoslovakia. Acta Sci. Nat. Acad. Sci. Bohewoslov, Bmo, $10(9)$ : 1.46.

Holden (M.J.) \& Green (J.). 1960 - The hydrology and plankton of the river Sokoto. J. Anim. Ecot., 29:65-84

Hynes (H.B.N.). 1970. - The ecology of running water. Liverpool Univ. Press, $555 \mathrm{p}$.
Juget (J.) Yi (B.J.), Roux (C.), Richoux (P.), Richardot-Coulet (M.) Reygrobellet (J.L.) \& Amoros (C.). 1979. - Structure et fonctionnement des ecosystemes du Haut-Rhône français. VII. Le complexe hydrographique de la Lóne des Pècheurs (un ancien méandre du Rhóne). Schueiz. Z. Hydrol, 41 (2) : 395-417.

Kieckhäfer. 1977. - Die gegenwärtigen Fischereigrundlagen det Binnenfischerei und Möglichkeiten einer gesteurten Nützung in Altrhein und Baggerseen. Der Fischwirt, 27 (7): 41.44 .

Mellors (W.K.). 1975. - Selective predation of ephippial Daphnia and the resistance of ephippial eggs to digestion. Eculogy, 56 (4) : 974-980.

Proctor (V.W.). 1964. - Viability of crustacean eggs recovered from Ducks. Ecology, 45 : 656-658.

Reygrobellet (J.L.), Mathieu (J.), Ginet (R.) \& Gibert (J.). 1981. Structure et fonctionnement des écosystèmes du Haut-Rhöne français. VIII. Hydrologie de deux stations phréatiques dont l'eau alimente des bras morts. Int. J. Speleol. 11 : 129.139.

Roux (A.L.) (ouvrage collectif publié sous la direction de). 1982. Cartographie polythematique appliquée à la gestion écologique des eaux : étude d'un hydrosystème fluvial : le Haut-Rhône français. Edit. CNRS, Centre Région. Publ. Lyon, $116 \mathrm{p}$.

Roux (A.L.). 1984. - The impact of emptying and cleaning reservoirs on the physicu-chemical and biological water quality of the Rhône downst ream of the dams. In A. Lillehammer \& S.J. Saltvein (ed.), Regulated Rivers, Universiteitsforlajet AS : $61-70$.

Stankovic (S.) \& Jankovic (D.). 1971. - Mechanismus der Fischproduktion im Gebiel des mittleren Donaulaufe. Arch. Hydro biol. (suppl. Donauforschung) suppl. 36 (4) : 299-305.

Straskraba (M.). 1965. - Contributions to the productivity of the littoral region of pools and ponds. I. Quantitative study of the littoral zooplankton of the rich vegetation of the backwater Labicko. Hydrobiologia, $26: 421.443$.

Straskraba (M.). 1967. - Quantitative study of the littoral zoo. plankton of the Polt ruba Backwater with an attempt to disclose the effect of fish. Rozpr. Ceskosl. Akad. Ved., mal. prirod. Ved. 77 (11): 7-34.

Vranovsky (M.). 1973. - Zur Bedeutung der Gewässer im Inundationsgebiet für das Plankton der Donau. Arbeitstagung der Internat. Arbeitsgem. Donauforschung. Bratislava, 16:6 p.

Vranovsky (M.). 1974 (a). - Zooplankton of the side arms system of Baka ahead of its confluence with the main stream and its importance for the forming of zooplankton of the river Danube (En slovaque, Res. Angl.). Biol. Prace, 20 (7) : 5.77.

Vranovsky (M.) 1974 (b). - Zur kenntnis der Verteilung, Biomasse und Drift des Zooplanktons im tschechoslowakish - ungaris. chen Donau-Abschnitt. Arch Hydrobiol, suppl., 44 (3) : 360-363.

Zivkovic (A.). 1968. - Das Zooplankton der jugoslawischen Donaust recke km 1424-86 I. Arch. Hydrabiol., suppl. 34 (suppl. Donauforschung) 155-167. 